\title{
CME Movement disorders (100624): self-assessment questionnaire
}

\author{
Edited by K Ray Chaudhuri and Tahseen A Chowdhury
}

\section{SAQs and answers are ONLINE for RCP fellows and collegiate members}

The SAQs printed in the CME section can only be answered online to achieve external CPD credits. Any comments should be sent in via email only: clinicalmedicine@rcplondon.ac.uk

\section{Format}

Candidates are asked to choose the best answer from the five possible answers. This best of five format is used in many medical examinations, however the questions are not intended to be representative of those used in the MRCP(UK) Part 1 or Part 2 Written Examinations.

\section{The answering process}

1 Go to www.rcplondon.ac.uk/SAQ

2 Log on using your usual RCP username and password

3 Select the relevant $\mathrm{CME}$ question paper

4 Answer all 10 questions by selecting the best answer from the options provided

5 Once you have answered all the questions, click on Submit

\section{Registering your external CPD credits}

Carrying out this activity allows you to claim two external CPD credits. These will be automatically transferred to your CPD diary, where you can review the activity and claim your points.

1 Which of the following symptoms is most predictive of future development of the clinical motor aspects of Parkinson's disease?
(a) anxiety
(b) depression
(c) gastrointestinal disorders
(d) hyposmia
(e) rapid eye movement behaviour disorder

2 In Parkinson's disease, during which of the following stages are non-motor symptoms present?

(a) advanced stage Parkinson's disease

(b) all throughout the disease course
(c) at diagnosis (early disease)
(d) on commencing therapy
(e) preclinical phase (prodromal Parkinson's disease)

3 What are the most common psychiatric disorders associated with Tourette syndrome?
(a) anxiety and obsessive compulsive disorder
(b) attention-deficit/hyperactivity disorder and anxiety
(c) attention-deficit/hyperactivity disorder and obsessive compulsive disorder
(d) depression and anxiety
(e) schizophrenia and depression

4 What is the most successful deep brain stimulation target in patients with dystonia?
(a) external globus pallidum
(b) internal globus pallidum
(c) sub-thalamic nucleus
(d) substantia nigra
(e) thalamus

5 What is the most common cause for restless legs syndrome (RLS)?
(a) anaemia
(b) diabetes
(c) idiopathic
(d) neuroleptics
(e) pregnancy

6 Which of the following treatment options is the most appropriate in a patient with severe RLS symptoms without any treatment at the moment?
(a) levodopa/carbidopa or levodopa/benzerazid
(b) lifestyle changes
(c) opioids
(d) pregabalin
(e) ropinirole

7 Approximately what percentage of patients met the criteria for minimal cognitive impairment (MCI) at the time of first diagnosis of Parkinson's disease?
(a) $5-10 \%$
(b) $20-50 \%$
(c) $70-90 \%$
(d) More than $90 \%$
(e) Unclear data 
8 Which drug was shown to be the most effective in treating psychosis in Parkinson's disease?

(a) amitriptyline

(b) clozapine

(c) nortriptyline

(d) olanzapine

(e) quietapine

9 Which of the following statements regarding the diagnosis of Parkinson's disease is correct?

(a) The diagnosis of Parkinson's disease is based on clinical symptoms. DaTSCAN ${ }^{\mathrm{TM}}$ is useful in the differential diagnosis between Parkinson's disease and atypical parkinsonism (eg MSA, PSP). Cardiac MIBG is useful in the differential diagnosis between Parkinson's disease and non-degenerative forms of parkinsonism such as essential tremor.

(b) The diagnosis of Parkinson's disease is based on the evidence of dopaminergic deficits assessed with DaTSCAN $^{\mathrm{TM}}$. Clinical symptoms may be only suggestive of disease but imaging is mandatory.

(c) The diagnosis of Parkinson's disease is based on clinical symptoms. DaTSCAN ${ }^{\mathrm{TM}}$ is useful in the differential diagnosis between Parkinson's disease and nondegenerative forms of parkinsonism such as essential tremor. Cardiac mIBG could be useful in the differential diagnosis between Parkinson's disease and atypical parkinsonism (e.g. MSA, PSP).

(d) The diagnosis of Parkinson's disease is based on the evidence of noradrenergic deficits assessed with cardiac mIBG. Clinical symptoms may be only suggestive of disease but imaging is mandatory.

(e) The diagnosis of Parkinson's disease is based on the combination of dopaminergic deficits assessed with a DaTSCAN ${ }^{\mathrm{TM}}$ and noradrenergic deficits assessed with cardiac mIBG. Clinical symptoms may be only suggestive of disease but imaging is mandatory.
10 Which of the following statements regarding the imaging of Parkinson's disease is correct?

(a) Striatal DaTSCAN ${ }^{\top M}$ binding is reduced in Parkinson's disease compared with atypical parkinsonism (MSA, PSP) and cardiac $\mathrm{mIBG}$ is reduced in atypical parkinsonism (MSA, PSP) compared with Parkinson's disease.

(b) Striatal DaTSCAN ${ }^{\top \mathrm{M}}$ binding is reduced in Parkinson's disease compared with non-degenerative forms of parkinsonism, such as essential tremor, and cardiac mIBG is reduced in Parkinson's disease compared with atypical parkinsonism (MSA, PSP).

(c) Striatal DaTSCAN ${ }^{\mathrm{TM}}$ binding is reduced in non-degenerative forms of parkinsonism, such as essential tremor, compared with Parkinson's disease and cardiac mIBG is reduced in Parkinson's disease compared with atypical parkinsonism (MSA, PSP).

(d) Striatal DaTSCAN ${ }^{\mathrm{TM}}$ binding is reduced in Parkinson's disease compared to atypical parkinsonism (MSA, PSP) and Cardiac MIBG is reduced in atypical parkinsonism (MSA, PSP) compared to non-degenerative forms of parkinsonism such as essential tremor.

(e) Striatal DaTSCAN ${ }^{\mathrm{TM}}$ binding is reduced in non-degenerative forms of parkinsonism, such as essential tremor, compared with Parkinson's disease and cardiac MIBG is reduced in atypical parkinsonism (MSA, PSP) compared with Parkinson's disease.

\section{CME Cardiology SAQ} Answers to the CME SAQ published in Clinical Medicine in June 2016

$\begin{array}{llllllllll}\text { Q1 } & \text { Q2 } & \text { Q3 } & \text { Q4 } & \text { Q5 } & \text { Q6 } & \text { Q7 } & \text { Q8 } & \text { Q9 } & \text { Q10 } \\ \text { (d) } & \text { (e) } & \text { (b) } & \text { (c) } & \text { (e) } & \text { (d) } & \text { (c) } & \text { (e) } & \text { (a) } & \text { (d) }\end{array}$

